\title{
Consenso Brasileiro de Monitorização e Suporte Hemodinâmico - Parte III: Métodos Alternativos de Monitorização do Débito Cardíaco e da Volemia*
}

\author{
Brazilian Consensus of Monitoring and Hemodynamic Support - Part III: \\ Alternative Methods for Cardiac Output Monitoring and Volemia Estimation
}

\author{
Guilherme Schettino, Ederlon Rezende, Ciro Leite Mendes, Álvaro Réa-Neto, Cid Marcos David, Suzana \\ Margareth Ajeje Lobo, Alberto Barros, Eliézer Silva, Gilberto Friedman, José Luiz Gomes do Amaral, Marcelo \\ Park, Maristela Monachini, Mirella Cristine de Oliveira, Murillo Santucci César Assunção, Nelson Akamine, \\ Patrícia Veiga C Mello, Renata Andréa Pietro Pereira, Rubens Costa Filho, Sebastião Araújo, Sérgio Félix Pinto, \\ Sérgio Ferreira, Simone Mattoso Mitushima, Sydney Agareno, Yuzeth Nóbrega de Assis Brilhante \\ pelo Painel de Especialistas do Consenso Brasileiro de Monitorização e Suporte Hemodinâmico.
}

\section{RESUMO}

JUSTIFICATIVA E OBJETIVOS: A interpretação do débito cardíaco e da pré-carga como números absolutos não traz grandes informações sobre a hemodinâmica do paciente crítico. Em contrapartida, a monitorização da resposta do débito cardíaco à expansão volêmica ou suporte inotrópico é uma ferramenta muito útil na unidade de terapia intensiva, quando o paciente apresenta algum sinal de má perfusão tecidual. Apesar do CAP ser considerado como "padrão-ouro" na avaliação destes parâmetros, foram desenvolvidas tecnologias alternativas bastante confiáveis para a sua monitorização.

MÉTODO: O processo de desenvolvimento de recomendações utilizou o método Delphi modificado para criar e quantificar o consenso entre os participantes. A AMIB determinou um coordenador para o consenso, o qual escolheu seis especialistas para comporem o comitê consultivo. Outros 18 peritos de diferentes regiões do país foram selecionados para completar o painel de 25 especialistas, médicos e enfermeiros. Um levantamento bibliográfico na MEDLINE de artigos na língua inglesa foi realizado no período de 1966 a 2004.

RESULTADOS: Foram apresentadas recomendações referentes à análise da variação da pressão arterial durante ventilação mecânica, débito cardíaco contínuo por contorno de pulso arterial, débito cardíaco por diluição do lítio, Doppler transesofágico, bioimpedância transtorácica, ecocardiografia e reinalação parcial de gás carbônico.

CONCLUSÕES: As novas e menos invasivas técnicas para medida do débito cardíaco, pré-carga e fluidoresponsividade apresentam adequada precisão e podem ser uma alternativa ao uso do CAP em pacientes graves. Unitermos: Consenso, Débito cardíaco, Monitorização Hemodinâmica, Pré-Carga, Prova de Volume, Recomendação.

\section{SUMMARY}

BACKGROUND AND OBJECTIVES: Cardiac output and preload as absolute data do not offer helpful information about the hemodynamic of critically ill patients. However, monitoring the response of these variables to volume challenge or inotropic drugs is a very useful tool in the critical care setting, particularly for patients with signs of tissue hypoperfusion. Although PAC remains the "gold standard" to measure cardiac output and preload, new and alternative technologies were developed to evaluate these hemodynamic variables.

METHODS: Modified Delphi methodology was used to create and quantify the consensus between the participants. AMIB indicated a coordinator who invited more six experts in the area of monitoring and hemodynamic support to constitute the Consensus Advisory Board. Twenty three physician and two nurses selected from different regions of the country completed the expert panel, which reviewed the pertinent bibliography listed at the MEDLINE in the period from 1996 to 2004.

RESULTS: Recommendations regarding the use of arterial pulse pressure variation during mechanical ventilation, continuous arterial pulse contour and lithium dilution cardiac output measurements, esophageal Doppler waveform, thoracic electrical bioimpedance, echocardiography and partial CO2 rebreathing for monitoring cardiac output and preload were created. 
CONCLUSIONS: The new and less invasive techniques for the measurement of cardiac output, preload or fluid responsiveness are accurate and may be an alternative to PAC in critically ill patients.

Key Words: Cardiac Output, Consensus, Fluid Challenger, Hemodynamic Monitoring, Recommendation.

\section{INTRODUÇÃO}

A interpretação do débito cardíaco e da pré-carga números absolutos não traz grandes informações sobre a hemodinâmica do paciente crítico. Em contrapartida, a monitorização da resposta do débito cardíaco à expansão volêmica ou suporte inotrópico é uma ferramenta útil na unidade de terapia intensiva, quando o paciente apresenta algum sinal de má perfusão tecidual ${ }^{1,3}$. Apesar do CAP ser considerado como "padrão-ouro" na avaliação destes parâmetros, foram desenvolvidas tecnologias alternativas bastante confiáveis para a sua apreciação. Serão discutidos outros métodos, que não o uso do cateter de artéria pulmonar (CAP), para a monitorização do débito cardíaco e da pré-carga nos pacientes instáveis hemodinamicamente ou para aqueles submetidos à cirurgia de grande porte.

\section{QUESTÃO: PODEMOS UTILIZAR A ANÁLISE DA PRESSÃO ARTERIAL DURANTE PROVA DE VOLUME?}

Recomendação: Sim. É uma técnica precisa para medir a responsividade do débito cardíaco à expansão volêmica. Depende da variação da onda de pulso arterial imposta pelo ventilador mecânico durante suporte ventilatório com pressão positiva.

Grau B2

Racional: Existem duas formas principais de analisar as variações da pressão arterial durante a ventilação mecânica com pressão positiva:

1. Variação da pressão sistólica ( $\triangle \mathrm{PS}$ ) entre as fases inspiratória (maior pressão sistólica) e expiratória (menor pressão sistólica). Na fase expiratória, a diminuição da pressão sistólica abaixo da linha de base é chamada de $\Delta$ down e fisiologicamente é o componente responsável por expressar a pré-carga do ventrículo direito - é esperado aumento do débito cardíaco após expansão volêmica quando o $\Delta$ down for maior que $5 \mathrm{mmHg}^{4}$.

2. Outra forma de avaliar a variação da onda de pulso arterial é através da variação da pressão de pulso $(\triangle \mathrm{PP})$, lembrando que pressão de pulso é a diferença entre a pressão sistólica e a pressão diastólica do ba- timento cardíaco imediatamente anterior. Este método consiste em expressar percentualmente a variação da pressão de pulso entre as fases inspiratória e expiratória do ciclo respiratório sob ventilação controlada, independentemente da pressão arterial basal, ou seja, estando o paciente hipotenso ou normotenso. O cálculo é simples e consiste em se dividir a diferença entre a pressão de pulso inspiratória (fisiologicamente maior) e a pressão de pulso expiratória (fisiologicamente menor) pela média das duas pressões de pulso, isto é:

$\Delta \mathrm{PP} \%=(\mathrm{PP}$ máxima $-\mathrm{PP}$ mínima $)$ (PP máxima + PP mínima)/2

Quando esta variação da pressão de pulso for maior ou igual a $13 \%$, têm-se um paciente com grande probabilidade de responder à expansão volêmica (sensibilidade de $94 \%$ e uma especificidade de $96 \%$ ), no qual a elevação do índice cardíaco em resposta a $500 \mathrm{~mL}$ de colóide será próxima ao valor obtido no cálculo do $\Delta \mathrm{PP}$ ${ }^{5}$. Estas análises predizem de forma acurada a responsividade à reanimação volêmica em pacientes sépticos e hipotensos (ou normotensos necessitando de drogas vasopressoras) e após grandes cirurgias ${ }^{6,7}$.

É uma técnica simples, que usa como recurso apenas um cateter intra-arterial e um monitor para registro das curvas de pressão arterial ${ }^{5}$.

Entretanto, cabe ressaltar que este método foi validado apenas para pacientes sépticos. Exige que o paciente esteja sob ventilação mecânica, sob sedação profunda e por vezes paralisado, de forma que possa ser utilizada uma modalidade ventilatória com volume controlado e sem que haja qualquer esforço inspiratório. Além disso, não pode ser utilizado com disritmias freqüentes ou persistentes (p. ex.: fibrilação atrial). Está padronizada para ser utilizada com PEEP de até $10 \mathrm{cmH}_{2} \mathrm{O}$ e volume corrente entre 8 e $10 \mathrm{~mL} / \mathrm{kg}^{5,8}$. Pacientes com disfunção de ventrículo direito podem apresentar falsos positivos e os pacientes com insuficiência cardíaca ou hipertensão pulmonar podem ter variações da pressão de pulso também falsamente positivas ${ }^{5}$.

\section{QUESTÃO: PODEMOS UTILIZAR O SISTEMA PCCO (PULSE CONTOUR CONTINUOUS CARDIAC OUTPUT) PARA MEDIDA DO DÉBITO CARDÍACO E RESPOSTA A INFUSÃO DE VOLUME?}

Recomendação: Sim. Este método permite a medida do débito cardíaco de forma precisa e contínua (batimento a batimento), quando comparado com a termodiluição 
pelo CAP, além da medida da pressão arterial sistêmica, o que favorece a detecção precoce de variações desses parâmetros quando houver mudança do estado hemodinâmico, assim como resposta à infusão de volume ou uso de drogas. A medida do volume diastólico final global e o cálculo do volume sanguíneo intratorácico, a variação da pressão de pulso e a variação do volume sistólico podem ser utilizados para avaliação precisa da pré-carga e da resposta à infusão de volume, com vantagem em relação à PVC e PAOP, por sofrer pouca interferência de variações da pressão intratorácica, abdominal e da função e complacência ventriculares ${ }^{2,9}$.

Grau B2

Racional: Nesta técnica, o volume sistólico e, onseqüentemente, o débito cardíaco, são calculados baseando-se na análise da área do contorno da onda de pulso aórtica obtida por um cateter posicionado preferencialmente na artéria femoral ou axilar, assumindose que a área determinada pela onda de pulso aórtica é proporcional ao volume sistólico ${ }^{10}$. A calibração da medida do débito cardíaco deve ser feita com o uso de algum indicador, que pode ser tanto a termodiluição transpulmonar como a curva de diluição do lítio. Após a calibração do sistema, o volume sistólico e o débito cardíaco são calculados de forma contínua e a cada batimento cardíaco.

A análise do contorno do pulso arterial associada à termodiluição transpulmonar permite também a medida do volume diastólico final global e a estimativa do volume sanguíneo intratorácico, além do cálculo da água pulmonar extravascular, parâmetros que podem ser utilizados para avaliação da pré-carga. Quando utilizado durante ventilação mecânica com pressão positiva, a variação do volume sistólico $(\Delta \mathrm{VS})$ pode ser utilizada para avaliação da pré-carga e como previsor de resposta para reposição volêmica em pacientes sépticos ou cirúrgicos ${ }^{11-14}$.

O Sistema PCCO (pulse contour continuous cardiac output) ${ }^{15}$ : utiliza o contorno da onda de pulso arterial para medida do volume sistólico e do débito cardíaco. A calibração do sistema para a medida do débito cardíaco é feita por termodiluição transpulmonar com indicador frio (solução fisiológica a $0,9 \%$ ou solução glicosada a $5 \%$, resfriada) administrado por cateter venoso central, sendo a variação de temperatura registrada pelo termístor localizado na linha arterial (preferencialmente em artéria femoral, porém descrita possibilidade de uso da artéria axilar). Os seguintes parâmetros podem então ser medidos ou calculados: volume sistólico, débito cardíaco, volume diastólico final global, assim como os seus valores indexados, como água pulmonar extravascular, além da pressão arterial sistólica, diastólica e média ${ }^{16}$. Quando utilizado durante ventilação mecânica com pressão positiva em modo controlado, dispõe-se também da variação dinâmica da pressão de pulso $(\Delta \mathrm{PP})$ e volume sistólico ( $\Delta \mathrm{VS})$ para auxílio na decisão para reposição volêmica.

Quanto às limitações do método, destaca-se a necessidade de cateter venoso central e linha arterial central, o que torna o método invasivo. A presença de grandes aneurismas aórticos pode superestimar os volumes medidos. Shunt intracardíaco, embolia pulmonar, ressecções pulmonares e disritmias podem prejudicar a acurácia do método. A boa precisão do método após calibração com a termodiluição se perde quando ocorrem importantes alterações na complacência vascular (vasoplegia ou vasoconstrição, por exemplo) fazendose necessária nova calibração ${ }^{16}$.

\section{QUESTÃO: PODEMOS UTILIZAR O DÉBITO CARDÍACO POR DILUIÇÃO DO LÍTIO PARA MEDIDA DO DÉBITO CARDÍACO E RESPOSTA A INFUSÃO DE VOLUME?}

Recomendação: Sim. É um método minimamente invasivo, sendo necessárias apenas punções venosa e arterial periférica. Permite a medida do débito cardíaco de forma precisa e contínua (batimento a batimento), quando comparado com termodiluição pelo $\mathrm{CAP}^{2,9}$, além de proporcionar a medida da pressão arterial sistêmica. O método favorece a detecção precoce de variações destes parâmetros, assim como permite a rápida avaliação da resposta clínica à infusão de volume ou uso de drogas ${ }^{2,9}$.

Grau B2

Racional: Esse sistema usa a diluição do lítio para a calibração do débito cardíaco. Nessa técnica, cloreto de lítio é injetado por um cateter venoso central ou periférico e a curva de concentração do lítio é medida por um eletrodo lítio-sensível conectado a uma linha arterial periférica. $O$ volume sistólico é calculado utilizando-se dados da onda de pulso arterial, sendo então possível a medida do débito cardíaco continuamente, a cada batimento cardíaco ${ }^{17-19}$. Quanto às limitações do método, deve-se destacar que não pode ser utilizado em pacientes recebendo lítio, assim como o uso de certos bloqueadores musculares pode interferir com a análise da curva de concentração do lítio. A presença de shunt intracardíaco inviabiliza o uso da técnica. A calibração, que pode ser necessária mais de uma vez ao dia, exige a coleta de pequena amostra de sangue. Quando é 
feita a opção pelo uso de acesso venoso periférico, o método não fornece informações diretas da pré-carga. Entretanto, quando utilizado durante ventilação mecânica com pressão positiva, em modo controlado, os dados de $\triangle \mathrm{PP}$ e $\Delta \mathrm{VS}$ podem ser utilizados para estimar a pré-carga e guiar a reposição volêmica ${ }^{17,18}$.

\section{QUESTÃO: PODEMOS UTILIZAR O DOPPLER TRANSESOFÁGICO PARA MEDIDA DO DÉBITO CARDÍACO E RESPOSTA A INFUSÃO DE VOLUME?}

Recomendações: Sim. É um método minimamente invasivo e tecnicamente simples que permite a monitorização em tempo real, batimento a batimento, do débito cardíaco, pré-carga e contratilidade cardíaca ${ }^{20-24}$. O aprendizado para a instalação do sistema e interpretação dos sinais obtidos é rápido. Existem poucas contra-indicações ao seu uso e o risco de eventos adversos é pequeno. $\mathrm{O}$ emprego deste método para ajustar a expansão volêmica, visando otimização do volume sistólico, mostrou-se benéfico em pacientes cirúrgicos e na sepse ${ }^{25-27}$.

Grau B2

Racional: O ultra-som Doppler da aorta permite a determinação do débito cardíaco de forma não invasiva, contínua e em tempo real.

O equipamento inclui transmissores de ultra-som e sensor posicionados na fúrcula esternal ou no esôfago, com janelas acústicas para a aorta ascendente ou descendente, respectivamente. O diâmetro da secção transversal da aorta é estimado por meio de um algoritmo ou medido por um segundo transdutor de ultra-som modo M. O método baseia-se na variação da freqüência da onda de ultra-som refletida pelo sangue que se desloca na aorta. A variação da freqüência é proporcional à velocidade do sangue. $\mathrm{O}$ volume sistólico deriva da velocidade de fluxo, tempo de ejeção e área da secção transversa da aorta. A avaliação da curva gerada pelo Doppler transesofágico permite estimar contratilidade (proporcional ao pico de velocidade ou amplitude) e pré-carga (proporcional à largura da base - FTc ou fluxo versus tempo corrigido) ${ }^{24}$.

A maioria dos estudos em animais e humanos demonstra que o método é preciso para a monitorização do débito cardíaco e da pré-carga ${ }^{20,22,23,28,29}$.

Entretanto, alguns fatores indutores de erros merecem ser mencionados: a posição do transdutor é crítica, visto que o ângulo de incidência do ultra-som acima de $20^{\circ}$ traz erro à medida; o diâmetro da aorta é uma variável crítica para o cálculo do débito cardíaco e sofre variação dinâmica, dependendo da pressão de pulso e da complacência do vaso; o fluxo sanguíneo é turbilhonar em situações como anemia, taquicardia e doença valvar aórtica; a determinação do débito na aorta descendente requer correção, pois corresponde à cerca de $70 \%$ do débito total, visto que $30 \%$ do débito dirigem-se ao crânio e membros superiores.

Outras limitações do método incluem a possibilidade de deslocamento da posição ideal da sonda, que exige ajustes intermitentes para garantir sinal ótimo e isso pode interromper a leitura. O sensor impõe desconforto semelhante ao de uma sonda gástrica de calibre semelhante e a posição correta para monitorização precisa exige imobilidade do paciente. Estes fatores tornam necessária a sedação. A possibilidade de traumatismo da mucosa esofágica exige cuidado na introdução do sensor, sobretudo quando houver associação com distúrbios da hemostasia. A otimização do sinal requer familiaridade (curva de aprendizagem) do operador com o método ${ }^{30}$. A introdução do sensor pode ser prejudicada em afecções do esôfago e o método não é indicado em pacientes com coarctação da aorta, dissecção aórtica e na presença de balão intra-aórtico.

\section{QUESTÃO: PODEMOS UTILIZAR AO BIOIMPEDÂNCIA TRANSTORÁCICA PARA MEDIDA DO DÉBITO CARDÍACO E AVALIAÇÃO DA VOLEMIA?}

Recomendação: Sim. Consiste em um método nãoinvasivo para medida do débito cardíaco, com boa concordância com a medida por termodiluição em voluntários normais e fornece monitorização contínua do débito cardíaco.

Grau B2

Racional: É uma técnica pouco precisa para mensuração do débito cardíaco e da volemia em pacientes críticos. Há vários fatores que reduzem a precisão das medidas, como: derrame pleural, derrame pericárdico, disritmias, sudorese intensa, edema, obesidade, ventilação mecânica, além de má precisão para estimar o débito cardíaco em medidas repetidas ${ }^{2,31,32}$.

\section{QUESTÃO: PODEMOS UTILIZAR O ECOCARDIOGRAMA PARA MEDIDA DO DÉBITO CARDÍACO E RESPOSTA A INFUSÃO DE VOLUME?}

Recomendações: Sim. É uma técnica precisa para a mensuração do débito cardíaco e mais ou menos pre- 
cisa para mensuração da volemia ${ }^{33-35}$.

Grau B2

Racional: Pode ser utilizado de forma não invasiva (transtorácico) ou minimamente invasiva (transesofágico), traz informações como fração de encurtamento miocárdico e sua resposta a inotrópicos, função diastólica ventricular, avaliação da pressão sistólica de artéria pulmonar ${ }^{35}$, o fluxo de ejeção do ventrículo esquerdo pode ser aferido. Pode-se ainda obter informações sobre as valvas, função contrátil segmentar e integridade anatômica das diversas estruturas cardíacas ${ }^{33}$. Usando-se o índice de variação da cava superior ( $\geq 36 \%$ ) com a ventilação mecânica, pode-se prever a resposta à expansão volêmica para aumento do débito cardíaco ( $\geq 10 \%)$ com sensibilidade de $90 \%$ e especificidade de $100 \%{ }^{36}$. Quanto às limitações, ressalta-se que é um método operador dependente; pode haver pacientes com janela anatômica que não permite a correta visualização do coração; há necessidade de um profissional capacitado; não tem boa correlação com o índice de trabalho do ventrículo esquerdo; e perde parte de seu valor, se o fluxo de ejeção do ventrículo esquerdo não for avaliado ${ }^{37}$.

\section{QUESTÃO: PODEMOS UTILIZAR A REINALAÇÃO PARCIAL DE GÁS CARBÔNICO (RBCO2) PARA MEDIDA DO DÉBITO CARDÍACO?}

Recomendação: Sim. Apresenta concordância satisfatória com a termodiluição, na ausência de lesão pulmonar ${ }^{38-40}$. Método não-invasivo e simples. Medidas tomadas automaticamente e em curtos intervalos. Aferições independentes da intervenção do examinador. O monitor disponível no mercado permite a obtenção de outros parâmetros respiratórios (p. ex: pressão e fluxo na via aérea, resistência de via aérea, complacência pulmonar, espaço morto, ventilação alveolar e produção de $\mathrm{CO}_{2}$ ), além da medida do DC.

Grau B2

Racional: A reinalação de gás carbônico $\left(\mathrm{RBCO}_{2}\right)$ consiste em um método de determinação do débito cardíaco por meio da aplicação do princípio de Fick na eliminação de $\mathrm{CO}_{2}$ :

$$
\mathrm{PCBF}=\frac{\mathrm{VCO}_{2}}{\mathrm{C}_{\mathrm{v}} \mathrm{CO}_{2}-\mathrm{C}_{\mathrm{a}} \mathrm{CO}_{2}}
$$

Onde : PCBF = fluxo sanguíneo pulmonar; $\mathrm{VCO}_{2}=$ eliminação de $\mathrm{CO}_{2} ; \mathrm{C}_{\mathrm{v}} \mathrm{CO}_{2}$ = conteúdo venoso misto de $\mathrm{CO}_{2} ; \mathrm{C}_{\mathrm{a}} \mathrm{CO}_{2}=$ conteúdo arterial de $\mathrm{CO}_{2}$.
Aplicada com e sem reinalação tem-se:

$$
\mathrm{PCBF}=\frac{\mathrm{VCO}_{2 \mathrm{~N}}}{\left(\mathrm{C}_{\mathrm{v}} \mathrm{CO}_{2 \mathrm{~N}}-\mathrm{C}_{\mathrm{a}} \mathrm{CO}_{2 \mathrm{~N}}\right)}=\frac{\mathrm{VCO}_{2 \mathrm{R}}}{\left(\mathrm{C}_{\mathrm{v}} \mathrm{CO}_{2 \mathrm{R}}-\mathrm{C}_{\mathrm{a}} \mathrm{CO}_{2 \mathrm{R}}\right)}
$$

Onde: $\mathrm{PCBF}$ = fluxo sanguíneo pulmonar; $\mathrm{VCO}_{2 \mathrm{~N}}=$ eliminação de $\mathrm{CO}_{2}$ sem reinalação; $\mathrm{VCO}_{2 \mathrm{R}}=$ eliminação de $\mathrm{CO}_{2}$ com reinalação; $\mathrm{C}_{\mathrm{v}} \mathrm{CO}_{2 \mathrm{~N}}=$ conteúdo venoso misto de $\mathrm{CO}_{2}$ sem reinalação; $\mathrm{C}_{\mathrm{v}} \mathrm{CO}_{2 \mathrm{R}}=$ conteúdo venoso misto de $\mathrm{CO}_{2}$ com reinalação; $\mathrm{C}_{\mathrm{a}} \mathrm{CO}_{2 \mathrm{~N}}=$ conteúdo arterial de $\mathrm{CO}_{2}$ sem reinalação; $\mathrm{C}_{\mathrm{a}} \mathrm{CO}_{2 \mathrm{R}}=$ conteúdo arterial de $\mathrm{CO}_{2}$ com reinalação.

Combinando para formar a Equação diferencial de Fick, temos:

$$
\begin{aligned}
& \mathrm{PCBF}=\frac{\mathrm{VCO}_{2 \mathrm{~N}}-\mathrm{VCO}_{2 \mathrm{R}}}{\left(\mathrm{C}_{\mathrm{V}} \mathrm{CO}_{2 \mathrm{~N}}-\mathrm{C}_{\mathrm{V}} \mathrm{CO}_{2 \mathrm{R}}\right)-\left(\mathrm{C}_{\mathrm{a}} \mathrm{CO}_{2 \mathrm{~N}}-\mathrm{C}_{\mathrm{a}} \mathrm{CO}_{2 \mathrm{R}}\right)} \\
& \frac{\Delta \mathrm{VCO}_{2}}{\Delta \mathrm{C}_{\mathrm{a}} \mathrm{CO}_{2}}=\frac{\Delta \mathrm{VCO}_{2}}{\mathrm{~S} \Delta \mathrm{ETCO}_{2}}
\end{aligned}
$$

Onde: $\Delta \mathrm{VCO}_{2}=$ diferença na eliminação de $\mathrm{CO}_{2}$ nas fases ${ }_{\mathrm{R}} \mathrm{e}_{\mathrm{N}} ; \Delta \mathrm{C}_{\mathrm{a}} \mathrm{CO}_{2}=$ diferença entre conteúdo arterial de $\mathrm{CO}_{2}$ nas fases ${ }_{\mathrm{R}} \mathrm{e}_{\mathrm{N}} ; \mathrm{S}=$ inclinação da curva de dissociação do $\mathrm{CO}_{2} ; \Delta \mathrm{ETCO}_{2}=$ diferença entre $\mathrm{CO}_{2}$ exalado ao final da exalação nas fases ${ }_{\mathrm{R}} \mathrm{e}_{\mathrm{N}}$.

Como as concentrações de $\mathrm{CO}_{2}$ no sangue venoso misto não sofrem mudanças significativas ao longo dos 50 segundos do período de reinalação, essas podem ser canceladas na equação matemática e, portanto, não são necessárias para o cálculo do PCBF. Isso permite que o PCBF seja obtido apenas com parâmetros nãoinvasivos. $\mathrm{O} \triangle \mathrm{ETCO}_{2}$ reflete o $\triangle \mathrm{PaCO}_{2}$.

O único monitor disponível atualmente no mercado inclui um dispositivo constituído de uma válvula adaptada ao circuito de reinalação e um sensor combinado de $\mathrm{CO}_{2}$ e fluxo ${ }^{41,44}$. Este conjunto é posicionado entre o tubo traqueal e o "Y" do circuito do ventilador. A alça de reinalação pode ser retraída ou expandida para otimizar o nível de reinalação. A $\mathrm{VCO}_{2}$ é calculada através da integração matemática do fluxo e sinais de $\mathrm{CO}_{2}$, medidos praticamente no mesmo ponto na via aérea do paciente, assegurando, desta forma, uma melhor precisão.

As variações de $\mathrm{VCO}_{2}$ e $\mathrm{ETCO}_{2}$ refletem somente as trocas gasosas que ocorrem nas áreas perfundidas e ventiladas dos pulmões. Isto fez necessário incluir no sistema um fator de correção para o fluxo de sangue desviado dos pulmões (shunt pulmonar). O computa- 
dor estima a fração de shunt com dados que lhe são fornecidos $\left(\mathrm{FiO}_{2}\right.$ e $\left.\mathrm{PaO}_{2}\right)$, saturação arterial de $\mathrm{O}_{2}$ (oximetria de pulso) e gráficos de iso-shunt de Nunn. $O$ DC é o resultado da soma do PCBF com o shunt calculado.

Entretanto, é um método que requer intubação traqueal e ventilação controlada com volume corrente constante, o que impede seu uso durante ventilação assistido-espontânea ou durante o desmame da ventilação mecânica. Ocorre também discordância com a termodiluição para medida do DC em pacientes com lesão pulmonar ${ }^{43,45}$, efeito que se acentua com o aumento da gravidade da lesão.

\section{PAINEL DE ESPECIALISTAS DO CONSENSO BRASILEIRO DE MONITORIZAÇÃO E SUPORTE HEMODINÂMICO}

Coordenador: Ederlon Rezende, Diretor do Serviço de Terapia Intensiva do Hospital do Servidor Público Estadual (São Paulo-SP);

Comitê Consultivo: Álvaro Réa-Neto, Professor do Departamento de Clínica Médica da UFPR, Chefe da UTI adulto do Hospital de Clínicas da UFPR, Diretor do CEPETI - Centro de Estudos e Pesquisa em Terapia Intensiva (Curitiba-PR); Ciro Leite Mendes, Coordenador da UTI adulto do Hospital de Emergência e Trauma Senador Humberto Lucena e Hospital Santa Paula, Médico da Rotina da UTI adulto do Hospital Universitário - UFPB (João Pessoa-PB); Fernando Suparregui Dias, Professor da Faculdade de Medicina da PUC de Porto Alegre, Chefe da UTI geral do Hospital São Lucas da PUC de Porto Alegre (Porto Alegre-RS); GuiIherme Schettino, Doutor em Medicina pela Faculdade de Medicina da USP, Médico Assistente da UTI Respiratória do HC da FMUSP, Médico Coordenador da UTI do Hospital Sírio Libanês (São Paulo-SP); Suzana Margareth Ajeje Lobo, Doutora de Medicina pela Universidade de São Paulo, Professora de clínica médica da Faculdade de Medicina de São José do Rio Preto, Coordenadora do Serviço de Terapia Intensiva do Hospital de Base - FAMERP (São José do Rio Preto-SP); Cid Marcos David, Presidente do Fundo de Educação e Pesquisa da Associação de Medicina Intensiva Brasileira, Doutor em Ciências Médicas, Professor Adjunto da UFRJ (Rio de Janeiro-RJ).

Painel de Especialistas: Alberto Barros, Médico Coordenador da UTI do Hospital Português de Recife (Recife-PE); Eliézer Silva, Doutor em Medicina, Médico Supervisor CTI Hospital Israelita Albert Einstein (São
Paulo-SP); Gilberto Friedman, Editor Chefe da Revista Brasileira de Terapia Intensiva; Médico do Hospital de Clínicas de Porto Alegre FAMED-UFRGS (Porto AlegreRS); José Luiz Gomes do Amaral, Doutor em Medicina, Chefe da Disciplina de Anestesiologia, Dor e Terapia Intensiva da UNIFESP (São Paulo-SP); Marcelo Park, Médico Assistente da Unidade de Terapia Intensiva Disciplina de Emergências Clínicas - HC-FMUSP (São Paulo-SP); Maristela Monachini, Doutora em Medicina, Médica Assistente da UTI do Hospital Sírio Libanês (São Paulo-SP); Mirella Cristine de Oliveira, Médica Coordenadora do Hospital do Trabalhador (CuritibaPR); Murillo Santucci César Assunção, Médico Coordenador Serviço de Terapia Intensiva do Hospital do Servidor Público Estadual (São Paulo-SP); Nelson Akamine, Médico Supervisor CTI Hospital Israelita Albert Einstein (São Paulo-SP); Patrícia Veiga C Mello, Médica Coordenadora da Disciplina de Emergência da Universidade Estadual do Piauí, Coordenadora da UTI do Hospital de Terapia Intensiva (Terezina-PI); Renata Andréa Pietro Pereira, Enfermeira Chefe do Serviço de Terapia Intensiva do Hospital do Servidor Público Estadual (São Paulo-SP); Rubens Costa Filho, Médico Coordenador CTI do PROCARDÍACO (Rio de Janeiro-RJ); Sebastião Araújo, Médico, Professor Doutor Assistente Departamento de Cirurgia da FCM-UNICAMP (Campinas-SP); Sérgio Félix Pinto, Médico Chefe da UTI do Hospital Universitário da UFMS (Campo Grande-MS); Sérgio Ferreira, Médico Chefe da UTI do Hospital Universitário Getúlio Vargas da UFAM (Manaus-AM); Simone Mattoso Mitushima, Enfermeira da UTI do Hospital Sírio Libanês (São Paulo-SP); Sydney Agareno, Médico Vice-Coordenador da UTI Geral do Hospital Português de Salvador (Salvador-BA); e Yuzeth Nóbrega de Assis Brilhante, Médica Coordenadora da UTI do Hospital da UNIMED João Pessoa (João Pessoa-PB).

\section{REFERÊNCIAS}

01. Pinsky MR - A rose by any other name: cardiac output. Crit Care Med, 2001;29:2021-2022.

02. Chaney JC, Derdak S - Minimally invasive hemodynamic monitoring for the intensivist: current and emerging technology. Crit Care Med, 2002;30:2338-2345.

03. Bendjelid K, Romand JA - Fluid responsiveness in mechanically ventilated patients: a review of indices used in intensive care. Intensive Care Med, 2003;29:352-360.

04. Tavernier B, Makhotine O, Lebuffe $\mathrm{G}$ et al - Systolic pressure variation as a guide to fluid therapy in patients with sepsis-induced hypotension. Anesthesiology, 1998;89:1313-1321.

05. Michard F, Boussat S, Chemla D et al - Relation between respiratory changes in arterial pulse pressure and fluid responsiveness in septic patients with acute circulatory failure. Am J Respir Crit Care Med, 2000;162:134-138.

06. Michard F, Teboul JL - Using heart-lung interactions to assess fluid res- 
ponsiveness during mechanical ventilation. Crit Care, 2000;4:282-289.

07. Kramer A, Zygun D, Hawes $\mathrm{H}$ et al - Pulse pressure variation predicts fluid responsiveness following coronary artery bypass surgery. Chest, 2004; 126:1563-1568.

08. Magder S - Clinical usefulness of respiratory variations in arterial pressure. Am J Respir Crit Care Med, 2004;169:151-155.

09. Malbrain MLNG, de Potter TJR - Cost-Effectiveness of Minimally Invasive Hemodynamic Monitoring. Berlin Heidelberg New York: SpringerVerlag; 2005.

10. Wesseling $\mathrm{KH}$, de Wit $\mathrm{B}$, Weber JAP - A simple device for the continuous measurement of cardiac output: its model basis and experimental verification. Adv Cardiovasc Phys, 1983:16-52.

11. Marx G, Cope T, McCrossan L et al - Assessing fluid responsiveness by stroke volume variation in mechanically ventilated patients with severe sepsis. Eur J Anaesthesiol, 2004;21:132-138.

12. Berkenstadt $\mathrm{H}$, Margalit $\mathrm{N}$, Hadani $\mathrm{M}$ et al - Stroke volume variation as a predictor of fluid responsiveness in patients undergoing brain surgery. Anesth Analg, 2001;92:984-989.

13. Reuter DA, Kirchner A, Felbinger TW et al - Usefulness of left ventricular stroke volume variation to assess fluid responsiveness in patients with reduced cardiac function. Crit Care Med, 2003;31:1399-1404.

14. Rex S, Brose S, Metzelder S et al - Prediction of fluid responsiveness in patients during cardiac surgery. Br J Anaesth, 2004;93:782-788.

15. Haller M, Zollner $\mathrm{C}$, Briegel $\mathrm{J}$ et al - Evaluation of a new continuous thermodilution cardiac output monitor in critically ill patients: a prospective criterion standard study. Crit Care Med, 1995;23:860-866.

16. Goraieb SM - O Método Volumétrico para a Avaliação Hemodinâmica. $1^{\text {a }}$ Ed, São Paulo: Editora Atheneu; 2004

17. Linton R, Band D, O'Brien T et al - Lithium dilution cardiac output measurement: a comparison with thermodilution. Crit Care Med, 1997;25:17961800.

18. Garcia-Rodriguez C, Pittman J, Cassell $\mathrm{CH}$ et al - Lithium dilution cardiac output measurement: a clinical assessment of central venous and peripheral venous indicator injection. Crit Care Med, 2002;30:2199-2204.

19. Jonas MM, Tanser SJ - Lithium dilution measurement of cardiac output and arterial pulse waveform analysis: an indicator dilution calibrated beat-by-beat system for continuous estimation of cardiac output. Curr Opin Crit Care, 2002;8:257-261.

20. Singer M, Clarke J, Bennett ED - Continuous hemodynamic monitoring by esophageal Doppler. Crit Care Med, 1989;17:447-452.

21. Cariou A, Monchi M, Joly LM et al - Noninvasive cardiac output monitoring by aortic blood flow determination: evaluation of the Sometec Dynemo-3000 system. Crit Care Med, 1998;26:2066-2072.

22. Valtier B, Cholley BP, Belot JP et al - Noninvasive monitoring of cardiac output in critically ill patients using transesophageal Doppler. Am J Respir Crit Care Med, 1998;158:77-83.

23. Bernardin G, Tiger F, Fouche R et al - Continuous noninvasive measurement of aortic blood flow in critically ill patients with a new esophageal echo-Doppler system. J Crit Care 1998;13:177-183.

24. Isola AM - Medida do Débito Cardíaco por Doppler Esofágico. O Método Volumétrico para a Avaliação Hemodinâmica. $1^{\mathrm{a}}$ Ed, São Paulo: Editora Atheneu; 2004.

25. Mythen MG, Webb AR - Perioperative plasma volume expansion reduces the incidence of gut mucosal hypoperfusion during cardiac surgery. Arch Surg, 1995;130:423-429.

26. Sinclair S, James S, Singer M - Intraoperative intravascular volume optimisation and length of hospital stay after repair of proximal femoral fracture: randomised controlled trial. BMJ 1997;315(7113):909-912.

27. Gan TJ, Soppitt A, Maroof M et al - Goal-directed intraoperative fluid administration reduces length of hospital stay after major surgery. Anesthesiology, 2002;97:820-826.

28. Baillard C, Cohen Y, Fosse JP et al - Haemodynamic measurements (continuous cardiac output and systemic vascular resistance) in critically ill patients: transoesophageal Doppler versus continuous thermodilution. Anaesth Intensive Care, 1999;27:33-37.

29. Dark PM, Singer M - The validity of trans-esophageal Doppler ultrasonography as a measure of cardiac output in critically ill adults. Intensive Care Med, 2004;30:2060-2066.

30. Lefrant JY, Bruelle P, Aya AG et al - Training is required to improve the reliability of esophageal Doppler to measure cardiac output in critically ill patients. Intensive Care Med, 1998;24:347-352.

31. Raaijmakers E, Faes TJ, Scholten RJ et al - A meta-analysis of three decades of validating thoracic impedance cardiography. Crit Care Med, 1999;27:1203-1213.

32. Summers RL, Shoemaker WC, Peacock WF et al - Bench to bedside: electrophysiologic and clinical principles of noninvasive hemodynamic monitoring using impedance cardiography. Acad Emerg Med, 2003;10:669-680.

33. Brown JM - Use of echocardiography for hemodynamic monitoring. Crit Care Med, 2002;30:1361-1364.

34. Vieillard-Baron A, Prin S, Chergui $\mathrm{K}$ et al - Hemodynamic instability in sepsis: bedside assessment by Doppler echocardiography. Am J Respir Crit Care Med, 2003;168:1270-1276.

35. Vieillard-Baron A, Prin S, Chergui K et al - Echo-Doppler demonstration of acute cor pulmonale at the bedside in the medical intensive care unit. Am J Respir Crit Care Med, 2002;166:1310-1319.

36. Vieillard-Baron A, Chergui K, Rabiller A et al - Superior vena caval collapsibility as a gauge of volume status in ventilated septic patients. Intensive Care Med, 2004;30:1734-1739.

37. Bouchard MJ, Denault A, Couture P et al - Poor correlation between hemodynamic and echocardiographic indexes of left ventricular performance in the operating room and intensive care unit. Crit Care Med, 2004;32:644-648.

38. van Heerden PV, Baker S, Lim SI et al - Clinical evaluation of the non-invasive cardiac output (NICO) monitor in the intensive care unit. Anaesth Intensive Care, 2000;28:427-430.

39. Binder JC, Parkin WG - Non-invasive cardiac output determination: comparison of a new partial-rebreathing technique with thermodilution. Anaesth Intensive Care, 2001;29:19-23.

40. Odenstedt $\mathrm{H}$, Stenqvist $\mathrm{O}$, Lundin S - Clinical evaluation of a partial CO2 rebreathing technique for cardiac output monitoring in critically ill patients. Acta Anaesthesiol Scand, 2002;46:152-159.

41. Capek JM, Roy RJ - Noninvasive measurement of cardiac output using partial CO2 rebreathing. IEEE Trans Biomed Eng, 1988;35:653-661.

42. Orr JA, Kofoed S, Westenskow D et al - A non-invasive cardiac output system using the partial rebreathing Fick method. J Clin Monit, 1996;12:464-465.

43. Gama de Abreu M, Winkler T, Pahlitzsch T etal - Performance of the partial CO2 rebreathing technique under different hemodynamic and ventilation/perfusion matching conditions. Crit Care Med, 2003;31:543-551

44. Jaffe MB - Partial CO2 rebreathing cardiac output--operating principles of the NICO system. J Clin Monit Comput, 1999;15:387-401.

45. Nilsson LB, Eldrup N, Berthelsen PG - Lack of agreement between thermodilution and carbon dioxide-rebreathing cardiac output. Acta Anaesthesiol Scand, 2001;45:680-685

Guilherme Schettino, Doutor em Medicina pela Faculdade de Medicina da USP, Médico Assistente da UTI Respiratória do HC da FMUSP, Médico Coordenador da UTI do Hospital Sírio Libanês (São Paulo-SP);

Ederlon Rezende, Diretor do Serviço de Terapia Intensiva do Hospital do Servidor Público Estadual (São Paulo-SP);

Ciro Leite Mendes, Coordenador da UTI adulto do Hospital de Emergência e Trauma Senador Humberto Lucena e Hospital Santa Paula, Médico da Rotina da UTI adulto do Hospital Universitário - UFPB (João Pessoa-PB);

Álvaro Réa-Neto, Professor do Departamento de Clínica Médica da UFPR, Chefe da UTI adulto do Hospital de Clínicas da UFPR, Diretor do CEPETI - Centro de Estudos e Pesquisa em Terapia Intensiva (CuritibaPR);

Cid Marcos David, Presidente do Fundo de Educação e Pesquisa da Associação de Medicina Intensiva Bra- 
sileira, Doutor em Ciências Médicas, Professor Adjunto da UFRJ (Rio de Janeiro-RJ). Fernando Suparregui Dias, Professor da Faculdade de Medicina da PUC de Porto Alegre, Chefe da UTI geral do Hospital São Lucas da PUC de Porto Alegre (Porto Alegre-RS);

Suzana Margareth Ajeje Lobo, Doutora de Medicina pela Universidade de São Paulo, Professora de clínica médica da Faculdade de Medicina de São José do Rio Preto, Coordenadora do Serviço de Terapia Intensiva do Hospital de Base - FAMERP (São José do Rio Preto-SP);

Alberto Barros, Médico Coordenador da UTI do Hospital Português de Recife (Recife-PE);

Eliézer Silva, Doutor em Medicina, Médico Supervisor CTI Hospital Israelita Albert Einstein (São Paulo-SP); Gilberto Friedman, Editor Chefe da Revista Brasileira de Terapia intensiva; Médico do Hospital de Clínicas de Porto Alegre FAMED-UFRGS (Porto Alegre-RS);

José Luiz Gomes do Amaral, Doutor em Medicina, Chefe da Disciplina de Anestesiologia, Dor e Terapia Intensiva da UNIFESP (São Paulo-SP);

Marcelo Park, Médico Assistente da Unidade de Terapia Intensiva - Disciplina de Emergências Clínicas - HC-FMUSP (São Paulo-SP);

Maristela Monachini, Doutora em Medicina, Médica Assistente da UTI do Hospital Sírio Libanês (São Paulo-SP);

Mirella Cristine de Oliveira, Médica Coordenadora do Hospital do Trabalhador (Curitiba-PR);
Murillo Santucci César Assunção, Médico Asistente da Disciplina de Anestesiologia, Dor e Terapia Intensiva da UNIFESP (São Paulo-SP);

Nelson Akamine, Médico Supervisor CTI Hospital Israelita Albert Einstein (São Paulo-SP);

Patrícia Veiga C Mello, Médica Coordenadora da Disciplina de Emergência da Universidade Estadual do Piauí, Coordenadora da UTI do Hospital de Terapia Intensiva (Terezina-PI);

Renata Andréa Pietro Pereira, Enfermeira Chefe do Serviço de Terapia Intensiva do Hospital do Servidor Público Estadual (São Paulo-SP);

Rubens Costa Filho, Médico Coordenador CTI do PROCARDÍACO (Rio de Janeiro-RJ);

Sebastião Araújo, Médico, Professor Doutor Assistente Departamento de Cirurgia da FCM-UNICAMP (Campinas-SP);

Sérgio Félix Pinto, Médico Chefe da UTI do Hospital Universitário da UFMS (Campo Grande-MS);

Sérgio Ferreira, Médico Chefe da UTI do Hospital Universitário Getúlio Vargas da UFAM (Manaus-AM);

Simone Mattoso Mitushima, Enfermeira da UTI do Hospital Sírio Libanês (São Paulo-SP);

Sydney Agareno, Médico Vice-Coordenador da UTI Geral do Hospital Português de Salvador (SalvadorBA);

Yuzeth Nóbrega de Assis Brilhante, Médica Coordenadora da UTI do Hospital da UNIMED João Pessoa (João Pessoa-PB). 